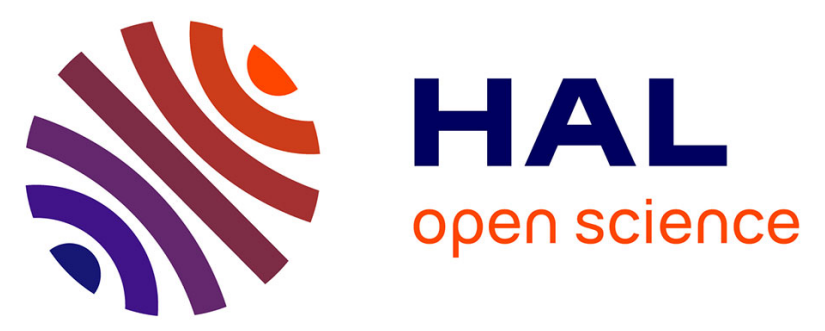

\title{
Observations And Experiments For The Definition Of A New Robotic Device Dedicated To CT, CBCT And MRI-Guided Percutaneous Procedures.
}

Antoine Pfeil, Laurent Barbé, Benoit Wach, Roberto Luigi Cazzato, Afshin Gangi, Pierre Renaud

\section{To cite this version:}

Antoine Pfeil, Laurent Barbé, Benoit Wach, Roberto Luigi Cazzato, Afshin Gangi, et al.. Observations And Experiments For The Definition Of A New Robotic Device Dedicated To CT, CBCT And MRI-Guided Percutaneous Procedures.. International conference with proceedings Observations and experiments for the definition of a new robotic device dedicated to CT, CBCT and MRI-guided percutaneous procedures, Jul 2018, Honolulu, United States. 10.1109/EMBC.2018.8512682 . hal02369393

\section{HAL Id: hal-02369393 \\ https://hal.science/hal-02369393}

Submitted on 18 Nov 2019

HAL is a multi-disciplinary open access archive for the deposit and dissemination of scientific research documents, whether they are published or not. The documents may come from teaching and research institutions in France or abroad, or from public or private research centers.
L'archive ouverte pluridisciplinaire HAL, est destinée au dépôt et à la diffusion de documents scientifiques de niveau recherche, publiés ou non, émanant des établissements d'enseignement et de recherche français ou étrangers, des laboratoires publics ou privés. 


\title{
Observations and experiments for the definition of a new robotic device dedicated to CT, CBCT and MRI-guided percutaneous procedures (Author manuscript, publisher version DOI: 10.1109/EMBC.2018.8512682)
}

\author{
Antoine Pfeil ${ }^{1}$, Laurent Barbé ${ }^{1}$, Benoit Wach ${ }^{1}$, Roberto Luigi Cazzato ${ }^{2}$, Afshin Gangi ${ }^{2}$ and Pierre Renaud ${ }^{1}$
}

\begin{abstract}
In this paper, we present the work achieved to define the robotic functionalities of interest for percutaneous procedures as performed in interventional radiology. Our contributions are twofold. First, a detailed task analysis is performed with workflow analysis of biopsies, one of the most frequent tasks, under three imaging modalities, namely CT, CBCT and MRI. Second, the functionalities of a robotic assistant are identified, and we analyze whether a single device can bring an added value during procedures in the three modalities while keeping the robotized workflow close to manual tasks, to minimize learning time and difficulty of use. Experimental analysis on CBCT is notably used to confirm the interest of the determined robotic functionalities.
\end{abstract}

\section{INTRODUCTION}

Interventional radiology (IR) is of great medical interest, given the potential achievable accuracy thanks to direct imaging during the medical procedure [1] [2]. Faster recovery time and the possibility of early and focal treatment are among the expected added values of IR [3] [4]. The number of percutaneous procedures, i.e. needle insertion tasks, is indeed increasing [5] [6] which shows the interest of the technique. Biopsies are becoming in particular more and more performed for the diagnostic of pathologies, in the context of personalized cancer care, with the goal to achieve a precise tumor cartography to adjust the subsequent treatment [7]. Of particular importance are biopsies on abdominal and thoracic organs, such as liver, kidney and lung, which represent one third of the overall cancer mortality worldwide [8], and on which we therefore focus.

Three imaging technologies offer high-quality images for such tasks. CT offers high resolution images. Cone Beam CT (CBCT) with robotized C-arm systems provide at the same time fluoroscopy and preliminary 3D volume reconstruction. In addition, integrated trajectory planning software now helps to obtain targeting accuracy similar to the one obtained with CT [9], with less X-ray exposure and easier access to the patient. MRI provides images with high contrast for soft tissues [10]. Major limitations however exist with the three imaging modalities. Exposure to X-rays raises a safety

\footnotetext{
*This work was supported by the INTERREG Upper Rhine program from the ERDF (European Regional Development Fund), SPIRITS project.

${ }^{1}$ Antoine Pfeil, Laurent Barbé, Benoit Wach and Pierre Renaud are with ICube Laboratory, Strasbourg, 67000 France a.pfeileunistra.fr

${ }^{2}$ Roberto Luigi Cazzato and Afshin Gangi are with the University Hospital of Strasbourg, France.
}

concern in the case of CT and CBCT, while for MRI, the small size of scanner gantry makes the patient access an issue and reduces the practitioner dexterity. For these reasons, robotic assistance can be of great interest in image-guided biopsies. Several systems have been introduced that solve technological bottlenecks related to X-ray compatibility [11] [12], some offer compatibility with X-ray and MR at the same time [13] [14]. However, most of the existing systems require automatic registration steps which do not exist in the manual procedure and are usually time-consuming and dependent on the imaging system. More importantly, none to our knowledge was built having in consideration procedures in the three modalities. As outlined in [15], task analysis is furthermore essential for an efficient design of the device and to our knowledge, no direct comparison of the three techniques has been presented up to now.

Our proposition is therefore to analyze closely the current manual practice for $\mathrm{CT}$, CBCT and MRI-guided tasks through procedure observations and interviews with the radiologists. Based on this analysis, we identify 1 . the difficulties of the manual procedure and the required functionalities to solve them, 2. how to use these functionalities in a detailed robotized procedure and 3 . the added-value in each modality. To confirm the identified robotic workflow, we finally propose an experimental evaluation with a proof of concept. In section II, analysis of biopsy procedures is introduced. Identification of robot functionalities is then derived in section III with associated workflow. Experimental analysis with CBCT is presented in section IV before concluding on the proposed robotic assistant definition in section $\mathrm{V}$.

\section{ANALYSIS OF BIOPSY PROCEDURES}

Our analysis needs to be generic to ensure usability of the robotic assistant. A multi-centric and multi-user analysis is thus performed, with 5 senior radiologists and 5 junior radiologists from the university hospitals of Strasbourg (France), Mannheim (Germany) and Basel (Switzerland) being involved.

\section{A. Breakdown of a manual biopsy procedure}

Biopsy involves the successive use of anaesthesia needles for anaesthesia, a coaxial needle for the path definition and a biopsy needle for the puncture. Eight steps are common to biopsy procedures in CT, CBCT and MRI, as represented 
in Table I, with in the chronological order:

1) Intra-operative planning. Image acquisition is followed by target identification and the needle path is defined by an entry point and a needle orientation.

2) Determination of the entry point on the patient.

3) Patient preparation. Disinfection is followed by the creation of a sterile environment with adhesive drapes.

4) Superficial anaesthesia. Topical anaesthesia using typically a $22 \mathrm{G}$ needle with a $10 \mathrm{~mm}$ insertion.

5) Deep anaesthesia. Anaesthesia along the path, using typically a $22 \mathrm{G}$ needle with a 20 to $40 \mathrm{~mm}$ insertion. Needle position, orientation and insertion are adjusted using visual monitoring with the images.

6) Placement of the coaxial needle. Before inserting the biopsy needle, the path is materialized by the placement of a coaxial needle. Its orientation is adjusted, then insertion is achieved to reach the target. $17 \mathrm{G}$ coaxial needles are typically used for a 50 to $70 \mathrm{~mm}$ insertion depth. Images are acquired to adjust orientation and insertion.

7) Biopsy puncture. Introduction of the biopsy needle in the coaxial needle, followed by a verification scan and the puncture.

8) End of procedure. After securing the samples, the coaxial needle is retracted.

\section{B. Difficulties related to the manual gesture}

According to our observations and interviews with radiologists, steps 5 and 6 are especially difficult to achieve in any imaging system, due to the accuracy needed for needle orientation and insertion. The nature of the difficulties is specific to each modality.

For CT procedures, no image can usually today be acquired during the needle manipulation, due to the radiologist's exposure to X-rays. The orientation adjustment is thus performed without direct image feedback, based on the radiologist's experience. This leads to an iterative procedure, with alternate needle manipulation and imaging for verification. This allows only small and time-consuming

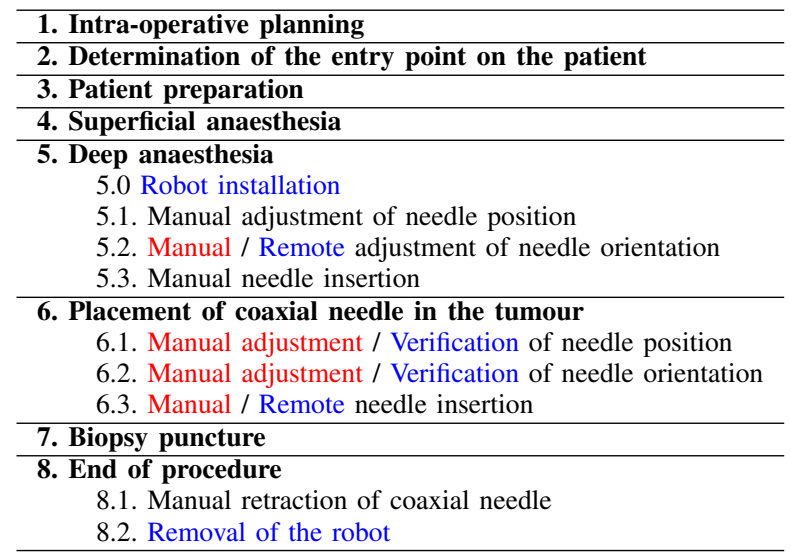

TABLE I: Workflow of a biopsy procedure. Color used to differentiate manual and robotic procedures (manual / robot). insertion steps, in order to ensure safety and for instance avoid vital structures such as blood vessels.

For CBCT-guided procedures, the X-ray exposure is lower than CT, so the radiologist can stay next to the patient during the procedure, with adequate protection. Nonetheless, the needle is remotely manipulated with a forceps to avoid direct hand exposure. This makes it difficult to realize fine motions. Needle insertion cannot be done in real-time without having the hands of the doctor exposed.

For MRI-guided procedures, a real-time imaging sequence can be used during the adjustment of needle orientation and insertion. However, given the small size of the scanner bore, typically 60 to $70 \mathrm{~cm}$, manipulation is very difficult, with limited accuracy during the fine motions required for the orientation task. Radiologist posture is also fatiguing, which has a negative impact on the precision.

\section{IDENTIFICATION OF ROBOT FUNCTIONALITIES, ASSOCIATED WORKFLOW AND ADDED-VALUE}

\section{A. Required robotic functionalities}

In order to solve the issues identified in section II, five functionalities appear of interest:

F1) Manual positioning of the needle at the entry point. Adjustment of needle position is quite straightforward. The robotic assistant therefore simply needs to allow manual modification of needle position.

F2) Remote control of needle orientation and insertion. Telemanipulation is of interest for several reasons. First, the assistant can be employed as an extension of the radiologist's hand. Needle manipulation can then remain intuitive and close to the manual practice. Radiologists stay in addition in charge of needle path adjustment, which is beneficial to safety. Since no automatic needle positioning is performed, robot registration is not needed, which saves time and makes the robot use easier. Finally, real-time images can be acquired during needle manipulation with the assistant. The real-time imaging is also beneficial for the safety of the patient, as the radiologist can immediately spot if there is a complication, and react adequately.

F3) Patient-mounted installation. Biopsy procedures are commonly performed under local anaesthesia. Patient motions can therefore occur during the procedure. Having the device patient-mounted allows to passively compensate for patient motion, to ensure patient safety.

F4) Information feedback on the transition between tissues. These haptic clues are necessary to provide information on the transitions between different tissues and organs.

F5) Online needle trajectory correction. During the procedure, the planned trajectory has sometimes to be slightly modified, due for instance to movements of the target. Trajectory adjustment will allow to reach the target, without a full retraction and insertion of the needle. 


\section{B. Associated procedure workflow}

The described functionalities are linked to the procedure workflow as shown in Table I. This workflow is very similar to the manual one as intended. More precisely, steps 1 to 4 are kept identical. During step 5, deep anaesthesia, one substep is introduced, which is installing the robot on the patient. The robotic assistant is then only used in substep 5.2. for orientation control. Indeed, a correct orientation is mandatory to ensure an efficient anaesthesia along the path of the coaxial needle to be inserted. The insertion can then be realized manually, since anesthesia does not need precise control of insertion depth. During the orientation task, the doctor adjusts the needle orientation remotely, using the real-time image feedback to achieve the planned trajectory.

As the anaesthesia needle and the coaxial needle follow the same path, the position at the entry point and the orientation achieved in steps 5.1. and 5.2. are used in step 6: needle position and orientation only need to be verified in step 6.1 and 6.2. The coaxial needle is then inserted in step 6.3. with the robotic assistant through remote control, with real-time feedback on the images. When a membrane is punctured, the system gives an information feedback to the doctor. If the needle deviates slightly from the pre-planned trajectory, the radiologist can adjust it to correctly reach the target.

When the tumour is entered, the biopsy is performed in the same manner than for the manual procedure. After the puncture, the coaxial needle is removed manually, as well as the robot.

\section{Expected Robotic Added-value}

First of all, as shown by Table I, the needle positioning and orientation tasks have only to be realized once with the robot, whereas they have to be done twice in the current manual procedure, in steps 5 and 6 . The robot functionalities and workflow also offer expected added-values which are specific to each of the three imaging modalities.

For CT, the use of the robot enables using CT-fluoroscopy during the procedure. This scanner functionality is today delicate to use because of X-Ray exposure of the doctors during direct manipulation. Remote manipulation offers the possibility to lower significantly doctor exposure to $\mathrm{X}$ rays. The real-time visualization of the needle during the orientation and insertion task is here a significant addedvalue with the robot.

For CBCT, the manipulation of the needle with the robot allows fine and stable needle motions, compared to the today tedious freehand manipulation with pliers. The X-ray exposure can also be reduced, as the radiologist is located further away from the X-Ray beam. Concerning insertion, the needle can be visualized in real-time, without exposure of the doctor, which is not possible with the current manual practice.

For MRI, the accessibility issue is solved by the telemanipulation, which can also improve dexterity and subsequently comfort during the procedure.

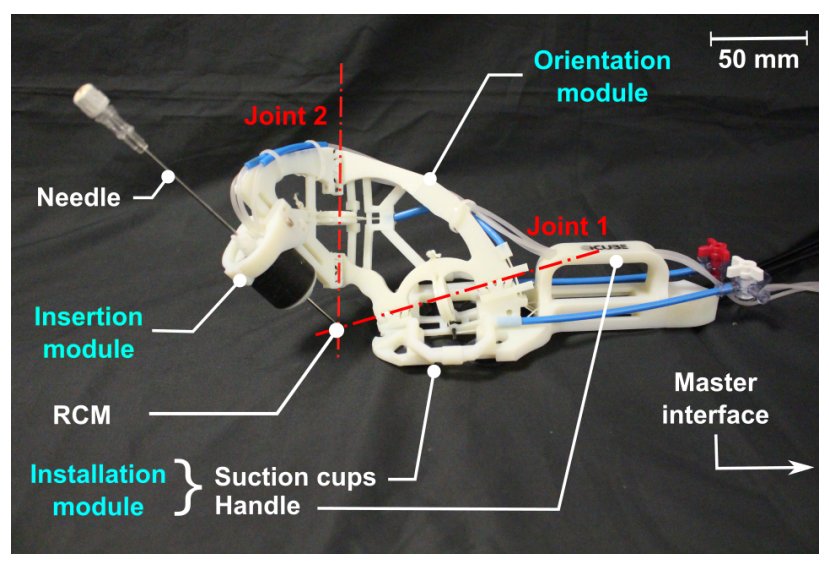

(a) Slave device for needle manipulation.

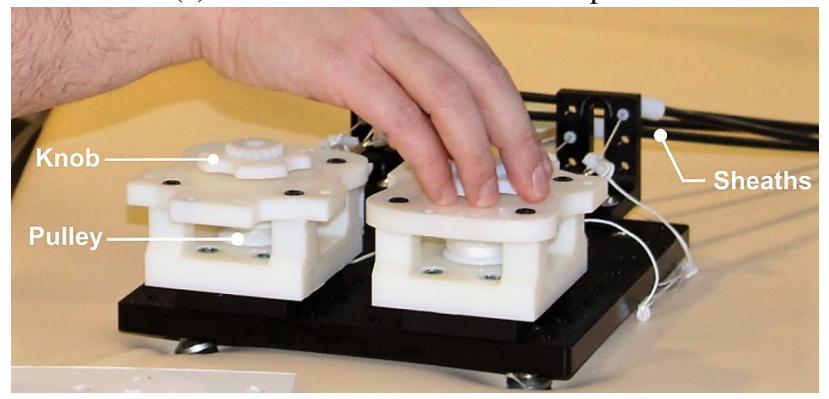

(b) Master interface for remote control of needle orientation.

Fig. 1: Global view of the proof of concept used for preclinical evaluation.

\section{EXPERIMENTAL ANALYSIS WITH CBCT}

In order to evaluate the impact of the robotic device on the medical workflow, an experimental analysis is performed with focus on functionalities F1), F2) and F3) as they are the most important for needle path control during steps 5 and 6 (see Table I). CBCT is selected as it is the easiest modality in terms of protocol for manual/robot comparison.

\section{A. Prototype presentation}

Assessment is performed with the robotic device depicted in Fig. 1. It is composed of a slave device and a master interface for remote needle manipulation. The slave device is based on three modules, as shown in Fig. 1a: one module for managing the needle position, one for needle orientation and one for needle insertion. The device is $110 \mathrm{~mm}$ high, $250 \mathrm{~mm}$ long and weighs less than $250 \mathrm{~g}$.

The slave device is positioned manually on the patient thanks to the handle, as shown in Fig. 2a. It is then maintained in position with four suction cups. The pressure in the suction cups can be easily modified so the fixation method is flexible in terms of patient morphology and fast for installation and removal.

The needle orientation module uses a 2-degrees of freedom serial spherical RCM (Remote Center of Motion) mechanism as introduced in [16]. It uses two revolute joints implemented with so-called HSC (Helical Shape Compliant) joints [17], the whole system being obtained using multimaterial additive 
manufacturing. Each joint is controlled remotely by means of a pair of antagonistic cables, wound up around a pulley on the master interface, as depicted in Fig. 1b. The operator can adjust the angle of each joint manually by turning two knobs driving the pulleys, as shown in Fig. 2b. Cable tension is adjusted using turnbuckles, cable sheaths are standard mechanical sheaths, of length equal to $1.10 \mathrm{~m}$. Needle can be oriented at \pm 45 degrees, following specs from [16].

The insertion module consists of a pneumatic actuator based on an inchworm principle [18] with a sequence of elementary motions for unlimited insertion. This allows to keep its length reduced to $45 \mathrm{~mm}$, with needle insertion speed of $1.4 \mathrm{~mm} / \mathrm{s}$, to achieve a $60-\mathrm{mm}$ insertion in less than 1 minute, which seems acceptable according to radiologists feedback. The actuator is able to generate forces up to $10 \mathrm{~N}$ as evaluated in lab conditions, which is sufficient to access abdominal organs [19]. The radiologist activates the needle insertion by pushing on a foot pedal, as shown in Fig. 2c.

\section{B. Protocol}

Experimental protocol is established to compare manual and robotic insertions. Two operators were involved to perform each 5 manual and 5 robotic insertions on a phantom based on $5 \%$ PVA mimicking the properties of liver tissue [20]. Insertion depths are equal to $60 \mathrm{~mm}$ with needle angulations of \pm 25 degrees with respect to the normal to the phantom surface. The imaging modality used was a CBCT system with path planning software $\left(\mathrm{XperCT}^{\circledR}\right.$ and XperGuide ${ }^{\circledR}$, Allura FD20, Philips Healthcare, the Netherlands). Each operator was given a 10-minute initial practice time before the trials.

Three criteria are used for procedure assessment. First, accuracy is measured using a 3D acquisition after each insertion. It is computed as the distance between the needle tip and the planned target point, with a measurement error of $\pm 0.5 \mathrm{~mm}$ due to the image resolution. Second, procedure duration is measured as the time elapsed between the start time of planning and the end of the procedure when the radiologist checks the final needle tip position. Third, Xray exposure is measured by a dosimeter fixed on the radiologist's wrist to assess level of exposure.

\section{Results}

Procedure as performed with the robotic device is illustrated in Fig. 2 with three phases for needle placement. No statistical difference between the two operators was observed for accuracy, procedure duration or X-ray dose. As a consequence, the mean and standard deviations of the three criteria reported in table II are computed after gathering the data sets.

The mean distance to target is equal to $3.8 \mathrm{~mm}$ for the manual and to $3.1 \mathrm{~mm}$ for the robotic insertions. Given the associated standard deviations reported in Table II, the accuracies of manual and robotic insertions do not significantly differ. An example of the needle image after a robotic insertion can be seen Fig. 3, showing that the needle is well aligned with the overlaid planned trajectory.

\begin{tabular}{l|c|c} 
Criterion & Manual & Robot \\
\hline Accuracy $(\mathrm{mm})$ & $\mathbf{3 . 8}(1.6)$ & $\mathbf{3 . 1}(0.9)$ \\
Procedure duration $(\mathrm{sec})$ & $\mathbf{3 1 5}(60)$ & $\mathbf{4 2 9}(71)$ \\
X-Ray exposure $(\mu \mathrm{Sv})$ & $\mathbf{1 . 1}(0.5)$ & $\mathbf{0 . 6}(0.4)$
\end{tabular}

TABLE II: Results of evaluation according to the three criteria. Mean values in bold letters, standard deviation between brackets.

Procedure duration is only increased by 2 minutes with use of the robotic device, whereas the initial practice time was very short. Radiologists reported a satisfactory feeling when manipulating the needle remotely with perception of robot dynamics due to the passive telemanipulation.

The major point is the X-Ray dose received by the radiologists, that is almost divided by two using the robot. This result is very interesting in terms of safety, and is as intended since the user manipulates more than $1 \mathrm{~m}$ away from the X-ray source during the orientation and insertion phases.

\section{CONCLUSION}

In this paper, definition of robotic functionalities and associated medical workflow have been introduced for biopsy procedures. The identified set of functionalities and the associated robotic workflow are compatible with procedures in CT, CBCT and MRI. It is shown that a single device providing simply remote control of needle orientation and insertion can bring an added value during procedures in the three imaging modalities while keeping the robotized workflow close to manual tasks, to minimize learning time and difficulty of use. Preliminary evaluation on CBCT shows that a significant X-ray dose reduction can potentially be achieved for the radiologists, while having a precision similar to the manual one and a minor impact on procedure duration.

Future work will now include the development of the robotic device, including the functionalities of information feedback on membrane puncturing and online trajectory correction. Polymer multimaterial additive manufacturing will be used for manufacturing, taking into account sterilization issues. We also consider to keep a direct passive orientation control for the safety it provides. Access to the patient and the impact of the suction cups will then also be investigated with tests including evaluation in CT and MRI.

\section{ACKNOWLEDGMENT}

The authors would like to thank the University Hospitals of Strasbourg (France), Mannheim (Germany) and Basel (Switzerland) and especially senior radiologists Dr. Rao, Dr. Koch, Dr. Garnon, Dr. Rathmann and Dr. Bilecen, for the procedure observations and interviews. Special thanks also go to intern Pierre Auloge and assistant radiology manipulator Emile Reeb for their participation in the experiments. This work was supported by the INTERREG Upper Rhine program from the ERDF (European Regional Development Fund), SPIRITS project. 


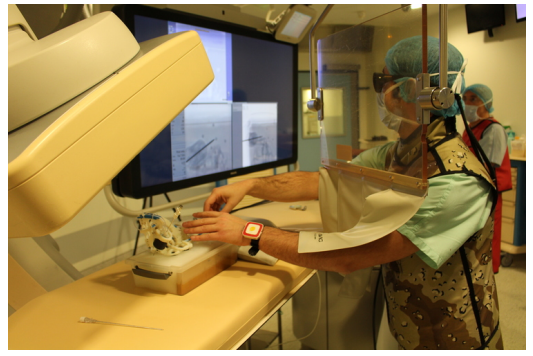

(a) Manual positioning

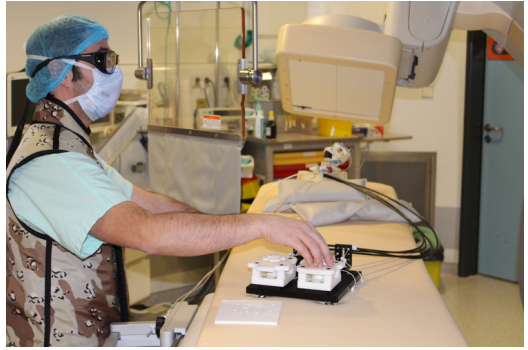

(b) Remote orientation

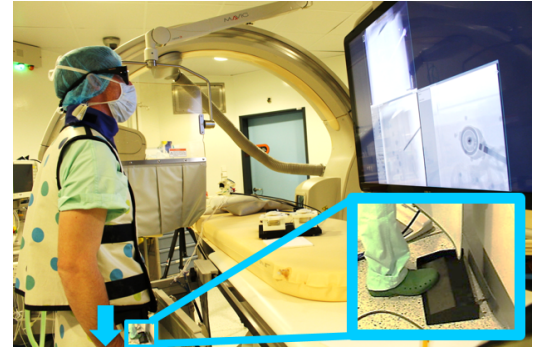

(c) Remote insertion

Fig. 2: Decomposition of the task with use of the robotic assistant.

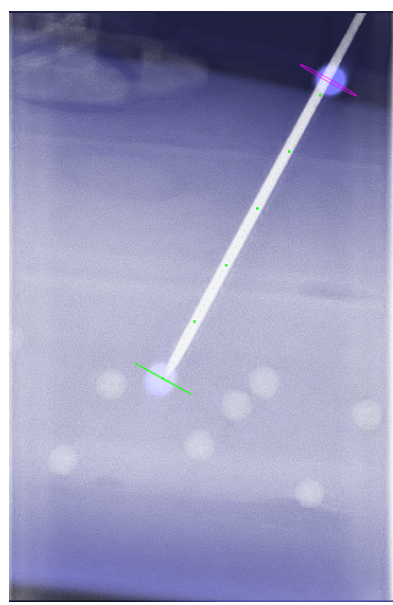

Fig. 3: Verification image after a robotic insertion. The green dots indicate the needle path as planned with the imaging modality software.

\section{REFERENCES}

[1] P. R. Mueller and E. van Sonnenberg. Interventional Radiology in the Chest and Abdomen. New England Journal of Medicine, 322(19):1364-1374, May 1990.

[2] J. L. Swischuk, F. Castaneda, J. C. Patel, R. Li, K.W. Fraser, T. M. Brady, and R. E. Bertino. Percutaneous Transthoracic Needle Biopsy of the Lung: Review of 612 Lesions. Journal of Vascular and Interventional Radiology, 9(2):347-352, March 1998.

[3] G. Pron, E. Mocarski, J. Bennett, G. Vilos, A. Common, M Zaidi, K. Sniderman, M. Asch, R. Kozak, M. Simons, C. Tran, and J. Kachura. Tolerance, Hospital Stay, and Recovery after Uterine Artery Embolization for Fibroids: The Ontario Uterine Fibroid Embolization Trial. Journal of Vascular and Interventional Radiology, 14(10):1243-1250, October 2003.

[4] M. L. Lessne, B.G. Oliverson, and P. Suhocki. Interventional Procedures for Global Health Radiology. In Radiology in Global Health, pages 181-188. Springer, New York, NY, 2014.

[5] M. E. Koran, A. J. Lipnik, J. C. Baker, F. Banovac, R. A. Omary, and D. B. Brown. Procedural Impact of a Dedicated Interventional Oncology Service Line in a National Cancer Institute Comprehensive
Cancer Center. Journal of the American College of Radiology: JACR, 13(9):1145-1150, September 2016.

[6] Technavio. Global Interventional Radiology Devices Market 20172021, 2017.

[7] A. L. Tam, H. J. Lim, I. I. Wistuba, A. Tamrazi, M. D. Kuo, E. Ziv, S. Wong, A. J. Shih, R. J. Webster, G. S. Fischer, S. Nagrath, S. E. Davis, S. B. White, and K. Ahrar. Image-Guided Biopsy in the Era of Personalized Cancer Care: Proceedings from the Society of Interventional Radiology Research Consensus Panel. Journal of Vascular and Interventional Radiology, 27(1):8-19, January 2016.

[8] Worldwide cancer mortality statistics, Cancer Research UK, May 2015.

[9] W. M. H. Busser, S. J. Braak, J. J. Ftterer, M. J. L. van Strijen, Y. L. Hoogeveen, F. de Lange, and L. J. Schultze Kool. Cone beam CT guidance provides superior accuracy for complex needle paths compared with CT guidance. The British Journal of Radiology, 86(1030):20130310, October 2013.

[10] N. V. Tsekos, A. Khanicheh, E. Christoforou, and C. Mavroidis. Magnetic resonance-compatible robotic and mechatronics systems for image-guided interventions and rehabilitation: a review study. Annual Review of Biomedical Engineering, 9:351-387, 2007.

[11] O. Piccin, L. Barbé, B. Bayle, M. de Mathelin, and A. Gangi. A Force Feedback Teleoperated Needle Insertion Device for Percutaneous Procedures. The International Journal of Robotics Research, 28(9):1154-1168, September 2009.

[12] O. Piccin, J. Sieffert, F. Schmitt, L. Barbé, L. Meylheuc, F. Nageotte, and B. Bayle. Design and characterization of a novel needle insertion tool. In IEEE International Conference on Biomedical Robotics and Biomechatronics (BioRob), pages 266-271, June 2016.

[13] N. Hungr, I. Bricault, P. Cinquin, and C. Fouard. Design and Validation of a CT- and MRI-Guided Robot for Percutaneous Needle Procedures. IEEE Transactions on Robotics, 32(4):973-987, August 2016.

[14] D. Stoianovici, C. Jun, S. Lim, P. Li, D. Petrisor, S. Fricke, K. Sharma, and K. Cleary. Multi-Imager Compatible, MR Safe, Remote Center of Motion Needle-Guide Robot. IEEE Transactions on Biomedical Engineering, 65(1):165-177, 2018.

[15] C. J. Walsh, N. C. Hanumara, A. H. Slocum, J.-A. Shepard, and R. Gupta. A Patient-Mounted, Telerobotic Tool for CT-Guided Percutaneous Interventions. Journal of Medical Devices, 2(1):011007, 2008.

[16] A. Bruyas, F. Geiskopf, and P. Renaud. Toward unibody robotic structures with integrated functions using multimaterial additive manufacturing: Case study of an MRI-compatible interventional device. In 2015 IEEE/RSJ International Conference on Intelligent Robots and Systems (IROS), pages 1744-1750, September 2015.

[17] A. Bruyas, F. Geiskopf, and P. Renaud. Design and Modeling of a Large Amplitude Compliant Revolute Joint: The Helical Shape Com- 
pliant Joint. ASME Journal of Mechanical Design, 137(8):085003085003-8, August 2015.

[18] K. El Bannan, B. A. Chronik, and S. P. Salisbury. Development of an MRI-Compatible, Compact, Rotary-Linear Piezoworm Actuator. Journal of Medical Devices, 9(1):014501-014501-7, March 2015.

[19] L. Barbé, B. Bayle, M. de Mathelin, and A. Gangi. In Vivo Model Estimation and Haptic Characterization of Needle Insertions. The International Journal of Robotics Research, 26(11-12):1283-1301, November 2007.

[20] S. Cournane, L. Cannon, J. E. Browne, and A. J. Fagan. Assessment of the accuracy of an ultrasound elastography liver scanning system using a PVA-cryogel phantom with optimal acoustic and mechanical properties. Physics in Medicine \& Biology, 55(19):5965, 2010. 